\section{EL ESTATUTO EPISTEMOLÓGICO DE LA BIOÉTICA}

\author{
Evandro Agazzi \\ Università degli Studi di Genova \\ Universidad Panamericana, México \\ ORCID iD: https://orcid.org/0000-0002-5131-7281 \\ evandro.agazzi@gmail.com
}

Cómo citar este artículo/Citation: Agazzi, E. (2019). El estatuto epistemológico de la bioética. Arbor, 195 (792): a500. https:// doi.org/10.3989/arbor.2019.792n2001

Recibido: 30 septiembre 2015. Aceptado: 28 abril 2016.

RESUMEN: La bioética no se presenta como una ciencia bien delimitada, sino más bien como un ámbito de cuestiones y problemas que se ha ido ampliando a partir de los años 70. En los inicios, la bioética abarcaba esencialmente la medicina y las biotecnologías, hoy incluye otros aspectos como el cuidado de los animales y la protección del medio ambiente, y muchos de sus temas conciernen a las políticas de salud y a la propuesta de normas legales. La novedad de la bioética, con respecto a la ética médica tradicional, se basa en el hecho de que el enorme progreso de las tecnologías en la práctica médica ha producido una gran cantidad de situaciones inéditas, en las que es posible y hasta necesario tomar decisiones para las cuales no existían normas en la ética tradicional. Esto depende también de la notable complejidad de las situaciones típicas de los debates bioéticos. Por estas razones, la bioética se presenta como un paradigma de lo que tiene que ser la ética en el contexto de una civilización tecnológica, es decir como la búsqueda de un punto de encuentro entre la tecnología y la conciencia moral. Si nos damos cuenta de esta característica fundamental, es posible reconocer en la bioética la presencia de un estatuto epistemológico propio, que consiste en la adopción del método interdisciplinar desde un enfoque sistémico y con capacidad de tomar en cuenta los fenómenos de la complejidad. De esta manera, la bioética conseguirá constituirse desde un punto de vista holístico que le permitirá relacionar los niveles más simples con los más complejos, así como aprovechar constructivamente las diferencias doctrinales y culturales que conlleva la globalización en nuestro tiempo.

PALABRAS CLAVE: Determinismo; complejidad; enfoque sistémico; holismo; ética de la tecnología; interdisciplinariedad.

\section{THE EPISTEMOLOGICAL STATUS OF BIOETHICS}

Copyright: (C) 2019 CSIC. Este es un artículo de acceso abierto distribuido bajo los términos de la licencia de uso y distribución Creative Commons Reconocimiento 4.0 Internacional (CC BY 4.0).

ABSTRACT: Bioethics, rather than a well-defined science, seems to more closely resemble a "field" of issues and problems that has been steadily expanding since the $70 \mathrm{~s}$. In the beginning, it essentially focused on medicine and biotechnology; but today includes other topics as well, such as animal care and environmental protection, health policies and bio-law. The recent and extremely significant progress in medical technology has produced several new moral problems that are not covered by traditional rules and general ethics. It is, however, possible, and even necessary, to take decisions regarding these new problems, and here resides the novelty and most valuable contribution of bioethics. The novelty also derives from the remarkable complexity of the more typical bioethical debates. For these reasons, bioethics could be presented as a paradigm of what ethics must constitute in the context of a technological civilization. It is a discipline that looks for a meeting point between technology and moral conscience. Given these fundamental characteristics, it is possible to sketch a special epistemological status for bioethics: this discipline adopts an interdisciplinary method based on a systemic approach as well as on the ability to cope with the phenomena of complexity. Thus, bioethics acquires a holistic point of view that allows for a connection between the simplest and most complex levels. Finally, we will show how bioethics takes advantage of the doctrinal and cultural differences brought about in our time by globalization.

KEYWORDS: Determinism; complexity; systemic approach; holism; ethics of technology; interdisciplinarity. 


\section{INTRODUCCIÓN. EL ÁMBITO DE LA BIOÉTICA}

Cuando se habla de estatuto "epistemológico" de algo se da por sentado que este algo es una ciencia o una disciplina científica y, por consiguiente, se admite implícitamente que de esta disciplina existe un concepto suficientemente claro y, en cuanto tal, expresable en una buena definición. De forma casi inmediata podemos encontrar el significado del término bio-ética considerando su etimología griega, en donde bíos significa vida, y la bio-ética, en consecuencia, resulta ser la ética de la vida. Sin embargo, este análisis nos llevaría probablemente a malinterpretar el significado que se le da realmente a la bioética hoy día, ya que en griego hay dos términos que nosotros traducimos por vida: uno es bíos y el otro es zoé. Bíos denota la vida entendida como 'forma de existencia' y en este sentido, por ejemplo, Aristóteles habla de "bíos theoretikós" para designar lo que más tarde se calificó como "vida contemplativa", mientras en otra ocasión habla del hombre como "zôon politikón", es decir como "animal político", para aludir a aquella especificidad de su naturaleza animal que consiste en el vivir en sociedad. Así las cosas, la bio-ética se caracterizaría como una disciplina que se interesa en determinar las condiciones para una "vida buena", y sería casi imposible distinguirla de la ética, entendida esta en su sentido más clásico y tradicional.

Pero el sentido de bíos en el griego antiguo no se corresponde con el sentido puramente científico-natural que la raíz bíos adquirió durante la modernidad, con el desarrollo de las ciencias de la vida. La raíz bíos apareció con su nuevo sentido esporádicamente en el transcurso del siglo XVIII, pero fueron Treviranus y Lamarck, en 1802, quienes usaron ya de manera explícita e independiente esta raíz en su nuevo sentido al acuñar el término biología. ${ }^{1}$ Ahora bien, tampoco esta precisión es de gran ayuda, ya que no se ve en qué sentido la ética podría relacionarse con la biología, siendo esta última una ciencia que se propone el estudio objetivo y riguroso de las características, propiedades y procesos que caracterizan a los seres vivos; estudio en el cual no caben juicios de valor ni, en particular, los juicios morales que nos autoricen a decir lo que en estos fenómenos naturales hay de bueno o de malo.

No es difícil reconocer aquí varios elementos de aquel debate acerca de la neutralidad de la ciencia que agitó la cultura occidental durante los años 19501960, y que acabó sin producir conclusiones por el hecho de no haber distinguido dos aspectos fundamentales de la ciencia, es decir, el hecho de que cons- tituye un sistema de saber y el hecho de que constituye también un sistema de actividades humanas. Mientras que la neutralidad con respecto a los juicios de valor es correcta considerando el saber científico, dicha neutralidad no existe si se considera el quehacer científico, que en cuanto tal se puede juzgar desde el punto de vista de diferentes valores y, en particular, desde el punto de vista de los valores éticos. Que esto realmente es así ya venía implícitamente reconocido en el mismo título de la obra de Treviranus, Biología, o sea filosofía de la naturaleza viva para los naturalistas y los médicos, título en el cual se mencionan, por un lado, los aspectos específicamente cognitivos de la biología (cultivados por los naturalistas) y, por otro lado, los aspectos pragmáticos representados por la utilización de estos conocimientos en la medicina. En aquel momento histórico a nadie le pasaba por la cabeza que se pudiese cuestionar éticamente el quehacer de los biólogos, simplemente porque se consideraba absolutamente bueno, tanto en su aspecto cognitivo (siendo un campo particular de la búsqueda de la verdad), como en su aspecto pragmático (siendo la medicina un ejemplo espléndido de uso de la ciencia para el bien del hombre).

Esto explica por qué, mientras el término biología se estableció desde el inicio del siglo XIX, el de bioética tardó en aparecer más de un siglo. Sin embargo, ya al final del siglo XIX empezaron a aparecer algunos problemas de tipo ético en el campo de la medicina, como consecuencia de su reiterado intento de convertirse en ciencia. Este esfuerzo exigía aprovechar los conocimientos proporcionados por la biología, así como los de la química y la física, para el diagnóstico y las terapias de las enfermedades, pero en un sentido más radical, con el propósito de darle a la medicina el corte específico de las ciencias naturales, es decir el uso del método experimental. El programa de una medicina experimental fue lanzado por Claude Bernard e ilustrado en su libro Introducción al estudio de la medicina experimental (Bernard, 1865). En esta obra, admirable por la claridad y elegancia de su estilo, encontramos una presentación muy detallada y rigurosa del método experimental en sus rasgos generales y en su aplicación al estudio de los diferentes tipos de "cuerpos", es decir de los "cuerpos brutos" y de los "cuerpos vivos". En ambos campos defiende un determinismo absoluto como fundamento del método experimental, cuya peculiaridad consiste en construir artificialmente una cierta situación de la cual debería deducirse necesariamente (según la hipótesis que se intenta averiguar) una situación causalmente bien determinada. Los cuerpos vivos de los que trata 
este libro son prácticamente los animales, y la ciencia fundamental que sirve para su estudio es la fisiología, en la cual el mismo Bernard había introducido el concepto innovador de ambiente interior, que se caracteriza por una serie de parámetros físico-químicos con valores bien determinados. La aplicación del método experimental en medicina consiste en considerar la situación de los cuerpos vivos, tanto en su estado normal como en su estado de enfermedad, mediante procedimientos comparativos como la "sección cadavérica" (es decir la autopsia), en la cual se basa la anatomía patológica, y la vivisección, cuyas características son detalladamente discutidas.

Lo que sorprende en este tratado de medicina es que no aparece una referencia al hombre y, por tanto, tampoco aparece el término paciente. Esto significa que, si la medicina quiere convertirse verdaderamente en una ciencia experimental, el paciente tiene que ser considerado como cualquier animal y, más radicalmente, como una "máquina viva" (machine vivante). Se podría pensar que esta es simplemente la consecuencia del hecho de que Bernard era un materialista duro, pero no es así. Su afán fundamental es que el científico experimental tiene que apegarse únicamente a los fenómenos que observa, formulando solo hipótesis directamente verificables, sin dejarse afectar por "doctrinas" generales, ya sean de tipo biológico, médico o filosófico. En cuanto tal, el experimentador no es ni materialista ni espiritualista, ni vitalista ni mecanicista, ni idealista ni positivista. No nos interesa analizar ahora si en realidad el mismo Bernard revela, en el trasfondo de su discurso, una filosofía y una metafísica implícita, es suficiente constatar que, en la conclusión de su obra, él declara explícitamente su fuerte aprecio por la filosofía entendida de modo particular. El capítulo IV de la tercera parte de esta obra se titula: "Los obstáculos filosóficos que se oponen a la medicina experimental", pero en el contenido de este capítulo, Bernard se declara contrario a los "sistemas filosóficos" y, al mismo tiempo, muy favorable al "espíritu filosófico". "La filosofía representa la aspiración eterna de la razón humana hacia el conocimiento de lo desconocido" (Bernard, 1865, p. 387) y, como tal, constituye la actitud fundamental de la misma ciencia y el resorte del progreso. Sin embargo, cuando un filósofo o una escuela pretende haber realizado un "sistema" completo que abarca o incluye todo lo conocible, se convierte en un obstáculo para el progreso (y esto vale también dentro de las diferentes ciencias). Por esta razón hay que promover un intercambio continuo entre ciencia y filosofía y ver en esta última la legítima aspiración a investigar cuestiones que van más allá de lo que las ciencias se plantean.

Gracias a estas precisiones de naturaleza metodológica, el propio Bernard dejaba abierto el camino para que el enfoque ético se asomara a la medicina científica. Es justo recordar que ya en el juramento hipocrático la medicina se enmarca dentro de un claro enfoque ético, pero en este libro Bernard considera la medicina hipocrática como prototipo de una medicina simplemente "descriptiva", capaz en el mejor de los casos de permitir previsiones acerca del decurso natural de una enfermedad, bien diferente de una medicina "experimental" que, mediante manipulaciones oportunas de las condiciones naturales, puede modificar el curso de la enfermedad. Por tanto, se puede entender en qué sentido el fisiólogo francés, al alejarse del modelo hipocrático, acabó ignorando también el marco ético de aquel modelo.

Sin embargo, la generalización del método experimental en medicina -y más precisamente en la práctica clínica- fue lo que muy pronto despertó la problemática ética. Fue así porque en dicha práctica se trata de experimentar sobre el ser humano, lo que impone espontáneamente a nuestra conciencia moral problemas que no percibimos de la misma forma cuando experimentamos sobre animales. De aquí nacen las reglamentaciones de la experimentación clínica contenidas en los códigos deontológicos de las organizaciones profesionales (no solo médicas). Dichos códigos se fueron estableciendo en muchos países hacia finales del siglo XIX y principios del XX. Representaron los primeros pasos de la ética médica moderna, al tiempo que fueron un preludio de la bioética. Su principal característica es que constituyen formas de auto-regulación cuyo objetivo general es asegurar el prestigio, la respetabilidad y el florecimiento de la profesión. Dictan, en particular, varias normas que conciernen al comportamiento del profesional con su cliente (en nuestro caso, del médico con el paciente). Sus normas vinculan solo a los miembros de la profesión, no tienen valor legal y no se fundamentan ni se inspiran en doctrinas o principios explícitamente reconocidos como éticos, sino en un marco de costumbres y principios comúnmente admitidos dentro de una determinada sociedad y en una determinada época histórica. Se pueden considerar, de esta manera, como preludio de la bioética y del bioderecho, en la medida en que han estimulado la investigación de los fundamentos éticos de sus normas y la traducción a prescripciones legales con valor general de algunas de ellas. 
Llegamos así al momento histórico en que nació explícitamente la bioética, es decir en que este término fue acuñado. Es conveniente traer aquí una historia ya bien conocida, pero también otra casi desconocida. La conocida, que se encuentra en la gran mayoría de las introducciones históricas de textos y artículos, dice que el término bioética fue creado por el bioquímico y oncólogo norteamericano Van Rensselaer Potter, quien lo usó en 1970 en un artículo y en 1971 en el propio título de un libro: Bioética. Puente hacia el futuro (Potter, 1971). A partir de ese momento, se difundió primero en los Estados Unidos y luego en Europa y en otras partes del mundo. Pero la verdad histórica es diferente: este término hizo su primera aparición en un artículo del teólogo y pastor protestante alemán Fritz Jahr en 1927, con un sentido muy amplio. El artículo, con el título Bio-Etica. Un análisis de la relación ética de los seres humanos con los animales y las plantas (Jahr, 1927) fue publicado por la prestigiosa revista científica Kosmos, precedido por otro artículo del mismo autor del año anterior con el título Las ciencias de la vida y la moral. Otros trabajos del mismo autor desarrollaron posteriormente sus ideas, que a día de hoy pueden considerarse realmente pioneras.

El concepto introducido por Jahr presenta una visión muy amplia de la relación moral entre el ser humano y el resto de los seres vivos, humanos y no humanos. Este autor desarrolló su "imperativo bioético" según un planteamiento de inspiración kantiana. Jahr apuntó a una ética respecto a los animales de experimentación, a la necesaria evaluación de las intenciones de la investigación científica y a un análisis de la difusión de la ciencia con el fin de hacer partícipe de la misma a la población general. Aunque no tuvieron repercusión debido a las circunstancias políticas y morales de su época, dominada por el nacionalsocialismo, los argumentos de Fritz Jahr fundamentalmente referidos a que una nueva ciencia y tecnología requieren nuevas reflexiones éticas y filosóficas, pueden considerarse como intuiciones pioneras de lo que debe ser no solo la bioética, sino también una ética a la altura de nuestro tiempo. Hay que decir, por otro lado, que el mismo Potter (quien muy probablemente ignoraba la existencia de los escritos de Jahr) también concibió la bioética en este sentido amplio, como una relación entre la acción del hombre y la preservación de un ambiente biológico adecuado para la supervivencia de la humanidad en las generaciones futuras.

Después de haber presentado la sucesión histórica correcta de los hechos, debemos sin embargo reconocer que la consideración de la bioética como una disciplina es reciente, existe desde hace menos de 50 años, y su identidad no está todavía bien asentada. Durante las primeras décadas de su historia ha sido considerada como un desarrollo de la ética médica (que cuenta con una larga historia); junto con los temas médicos, se admitían dentro de su ámbito también los problemas éticos suscitados por las biotecnologías. Por consiguiente, era normal definir la bioética como el estudio de los problemas éticos suscitados por las ciencias biomédicas y sus aplicaciones. Ámbitos como los de la ética animalista o los de la eco-ética (es decir la ética del medioambiente) eran considerados por muchos como digresiones casi arbitrarias, o en el mejor de los casos como campos de discusiones, a veces interesantes, que apasionaban a un grupo muy limitado de adeptos y aficionados. Se trataba de una situación de hecho, ligada a la circunstancia de que las primeras instituciones que surgieron con el propósito explícito de promover la bioética, de organizar las investigaciones en este campo, de darle una estructura de tipo académico, de promover publicaciones y hasta tratados y enciclopedias de bioética, ponían el acento sobre las ciencias biomédicas, y el público al cual se dirigían preferentemente era el de las profesiones médicas e instituciones relacionadas con el cuidado de la salud. Nos referimos al Hastings Center y al Kennedy Institute, apoyado este último por la Universidad de Georgetown. Ambos fueron formalmente fundados, como transformación de instituciones pre-existentes, en 1971. Esta delimitación temática se conservó de manera esencial en los centros de bioética que pocos años después surgieron en Europa y en otras partes del mundo; no es por mera casualidad que la mayoría de ellos se encontraban, y todavía se encuentran, orgánicamente relacionados con hospitales, clínicas, facultades y escuelas de medicina.

Cuando hablamos de una cuestión de hecho no queremos banalizar el asunto, ya que ha sido una práctica tradicional la de definir una cierta ciencia tomando en cuenta lo que hacen concretamente los que se dedican a ella. ${ }^{2}$ Sin embargo, es legítimo preguntarse también si hay razones de principio que justifiquen esta caracterización. Sin que llegue a ser una verdadera razón de principio, podemos decir que esta referencia privilegiada de la bioética a las ciencias biomédicas se explica también por el intento de la bioética de presentarse ella misma como una ciencia, como un campo de investigación riguroso, en el cual se comparan argumentos y que, por tanto, busca sus credenciales de cientificidad concentrando sus objetos de reflexión en lo que se dice y hace en ámbitos científicos serios 
y reconocidos. Y como bío refiere a vida, inmediatamente se sigue que de lo que la bioética se ocupa es de las cuestiones éticas que surgen dentro de las ciencias y tecnologías de la vida, es decir en las ciencias biológicas y médicas.

\section{2. ¿POR QUÉ SE REQUIERE LA INTRODUCCIÓN DE UN TÉRMINO NUEVO?}

Las razones que acabamos de exponer aclaran tal vez cómo fue produciéndose la situación histórica que determinó la delimitación del campo de estudio (del ámbito) de la bioética. Sin embargo, mientras las circunstancias ofrecen una respuesta a esta pregunta, abren una segunda cuestión: si es verdad que la bioética es fundamentalmente un desarrollo, una expansión natural de la ética médica tradicional, ¿qué necesidad teníamos de inventar un nuevo término para designarla? Si la bioética no quiere ser una simple expresión de moda para denotar algo ya existente, sino el nombre de una disciplina auténticamente nueva, hasta de una nueva ciencia, es indispensable indicar claramente en qué consiste su novedad, y esto resultaría más patente si fuera posible indicar alguna diferencia con respecto a la ética médica tradicional. Algunos autores, como por ejemplo Maurizio Mori (2002), han intentado este tipo de discriminación, afirmando que existe una verdadera diferencia "de principio": mientras la ética médica tradicional aceptaba como principio ético fundamental el de la sacralidad de la vida, la bioética, por el contrario, se funda en el principio de la cualidad de la vida. Esta distinción no parece muy sólida: podría servir para caracterizar diferentes teorías éticas (actuales y también tradicionales), pero no como criterio para discriminar entre la ética médica tradicional y la bioética actual, ya que, en primer lugar, ambas perspectivas están presentes en los debates bioéticos actuales y, en segundo lugar, porque en la misma ética médica tradicional (que sin duda reconocía la superioridad del principio de la sacralidad de la vida), no se desconocía tampoco la validez del principio de la cualidad de la vida. Tan es verdad, que se admitía que los dolores intolerables de un enfermo terminal se deben aliviar con tratamientos adecuados, aunque estos tengan como consecuencia no querida una abreviación de la vida del paciente (lo cual es moralmente distinto de provocarle deliberadamente la muerte para que "deje de sufrir"). Como los debates bioéticos actuales atestiguan ampliamente que estos dos principios, lejos de estar en conflicto, son complementarios, uno de los desafíos principales del pensamiento bioético es el de proponer soluciones que los hagan compatibles, algo que los avances de la medicina y de las técnicas médicas hacen hoy más factible.
Como última observación anotemos que los conflictos entre estos dos principios que marcan el debate bioético actual inciden en la casi totalidad de las prácticas médicas, es decir que caben dentro del horizonte de la ética médica. Por lo tanto, no se ve cómo el tomarlos en consideración nos permitiría superar el horizonte de la ética médica para entrar en el "nuevo" dominio de la bioética.

Entonces ¿no hay novedad? Sí la hay, y es posible caracterizarla de manera más concreta y sin afectar a los grandes principios éticos. La novedad consiste en el hecho de que la tarea esencial de la ética -es decir, la de indicar cuál es la elección correcta ante diferentes conductas posibles en una determinada situación- se ha convertido en una empresa muy difícil en muchas situaciones totalmente inéditas que se presentan como consecuencia del progreso científico y tecnológico. Hay que entender bien en qué consiste el aspecto "inédito": no se trata simplemente de la complejidad a veces asombrosa de muchos aparatos y procedimientos que se utilizan, por ejemplo, en el campo de la medicina y de la cirugía, y que permiten alcanzar resultados antes impensables. Se trata, más bien, del hecho de que, como consecuencia de estos resultados, se presentan situaciones en las cuales el hombre puede elegir, optar, decidir y que, por lo tanto, caen automáticamente bajo el juicio moral, mientras que antes no se daban tales situaciones y, por esta simple razón, no podían ser contempladas por la ética. Por ejemplo, algunas décadas atrás la esterilidad de una pareja (entendida como imposibilidad de engendrar hijos dentro de la misma) se intentaba curar tratando ciertas disfunciones tanto del varón como de la mujer. Si tales disfunciones no se podían curar, o si la pareja resultaba estéril a pesar de todo, la medicina resultaba inoperante y la única solución posible consistía en recurrir al instrumento legal de la adopción. Hoy sabemos que una pareja puede conseguir un hijo a pesar de ser médicamente estéril, sometiéndose a alguna de las técnicas biomédicas disponibles y, por lo tanto, puede decidir si utilizarlas o no. Incluso, en caso de querer utilizarlas, puede decidir cuál prefiere. Todo el mundo sabe que este es uno de los capítulos fundamentales de la bioética, el de la reproducción medicamente asistida, y parece evidente que no se puedan proponer normas morales a este propósito sin un conocimiento detallado de las situaciones reales, que son totalmente inéditas con respecto a la reproducción natural anteriormente conocida y regulada por la ética. 
Aparentemente se podría decir que, sin embargo, estas nuevas situaciones se pueden y hasta se deben tratar aplicándoles los principios fundamentales y las normas de la ética tradicional, y que no se necesita una nueva ética para abordarlas. En cierto sentido es así, pero a condición de reparar en que la propia ética no se reduce a un conjunto estático e inmutable de principios, métodos y preceptos formulados en una determinada doctrina, sino que ella misma tiene que ser dinámica y articularse, profundizarse, explicitarse, según los distintos contextos culturales en los que se sitúa. Y, aun así, habría que considerar otro aspecto que justifica la novedad de la bioética: se trata de la naturaleza compleja de las situaciones inéditas de las que estamos hablando. Con ello nos referimos en particular al hecho de que la acción de cada individuo involucrado está profundamente relacionada y condicionada por la presencia de otros individuos. Esto conlleva un enfoque relativamente ajeno a la perspectiva de la ética tradicional, que era sobre todo una ética de la acción individual. Para profundizar en este punto es oportuno discutir primero cómo este tipo de complejidad influye sobre la naturaleza misma de la bioética en cuanto ciencia.

La noción de complejidad posee diferentes matices de significado, tanto en el discurso ordinario como en el científico. En el ámbito pre-científico, el significado de complejo tiende a ser visto como sinónimo de dificil o complicado, mientras que en el discurso científico no existe una única caracterización de la complejidad. Cada definición depende del contexto en el que nace, incluso cuando es adoptada con propósitos de generalidad (como ocurre, por ejemplo, en el famoso Santa Fe Institute for Complexity Research, activo en New Mexico, Estados Unidos).

Entre los principales significados, se pueden destacar dos que se refieren esencialmente a la noción de problema o de objeto complejo. La complejidad computacional se refiere a la búsqueda de la solución de un problema que se pueda conseguir mediante un programa de cálculo automático. Se han propuesto diferentes medidas de la complejidad computacional. La más conocida mide la complejidad como la dimensión de la formulación del problema computacional en cuestión, y se expresa como la longitud del programa más pequeño que, aplicado en un ordenador universal (máquina de Turing), ofrece la solución del problema. Según esta definición, llamada de Kolmogoroff y Turing, son simples los problemas o los objetos muy regulares, o bien los que son totalmente casuales, ya que en ambos casos hay poco o nada que describir, mientras que son complejos los problemas y objetos cuya descripción es larga. En la noción más completa de complejidad computacional se añade a las condiciones de Kolmogoroff también la consideración del tiempo de cómputo y del espacio de memoria que es necesario utilizar.

Un concepto muy diferente de complejidad ha surgido del estudio de los sistemas dinámicos alejados del estado de equilibrio, del caos determinístico y de la emergencia de comportamientos nuevos debidos a la interacción entre las partes de un sistema. Este concepto ha sido llamado complejidad de las redes de interacción, en las cuales ocupa un lugar central la nolinearidad. Omitiendo su presentación técnicamente matemática, podemos sintetizar su consecuencia intuitiva más significativa diciendo que expresa la imposibilidad de descomponer un problema en sub-problemas para luego recomponer las soluciones parciales consiguiendo así la solución del problema inicial. Este último procedimiento había sido enunciado por Descartes como la segunda regla en la segunda parte del Discurso del método, y su aplicación ha constituido el modelo de la investigación científica moderna, basada en lo que se define como método analítico.

Se suele indicar que el preludio de la nueva perspectiva está en un famoso artículo de Poincaré sobre el problema de los tres cuerpos (Poincaré, 1890). Es interesante porque muestra que la idea de una previsibilidad exacta de los fenómenos naturales, fundada en el determinismo universal, en el que Claude Bernard creía firmemente, no es científicamente defendible. Diremos, por simplicidad, que un sistema $\mathrm{S}$ evoluciona según un proceso lineal si, dado el valor del estado inicial del sistema con una aproximación $\varepsilon$, se puede calcular su estado final después de cualquier tiempo con la misma aproximación. Si esto no ocurre, es decir, si el estado final puede tener un valor muy diferente de lo que se podía esperar, el proceso se llama no-lineal, terminología que depende del tipo de ecuaciones diferenciales que se aplican. Ahora bien, se puede probar matemáticamente que los resultados imprevisibles y nuevos se dan, no solo cuando hay interacciones no-lineales entre sistemas no-lineales, sino también cuando hay relaciones no-lineales entre sistemas lineales. Dicho más resumidamente: el resultado de interrelaciones entre procesos determinísticos es determinístico solo en casos muy excepcionales. Traduciendo este discurso abstracto a términos más concretos, podemos decir que si varios sistemas, caracterizados cada uno de ellos por sus propiedades típicas, se encuentran en correlación, el resultado glo- 
bal será un sistema con propiedades nuevas, no presentes en ninguna de sus partes.

Este breve análisis de la complejidad de las redes de interacciones nos ha permitido detectar un nivel intermedio entre el orden completo y el completo desorden, es decir entre el determinismo rígido, que era el ideal de la ciencia clásica claramente presente en el libro de Bernard, y el caos total. No es algo secundario que, para esta caracterización más adecuada de la complejidad, hayamos tenido que usar algunas veces el término sistema: se trata de que el punto de vista sistémico, es decir, la teoría general de sistemas, se ofrece como un instrumento esencial para alcanzar el resultado que buscamos.

\section{EL PUNTO DE VISTA SISTÉMICO}

El tratamiento matemático de la complejidad que hemos considerado hasta ahora, y que alcanza su punto de mayor interés en la teoría de la no-linealidad, ya ha contribuido a deshacer el mito del determinismo universal, sobre todo si nos apoyamos en la expresión de la no-linealidad que, siguiendo el planteamiento geométrico de Poincaré, representa el decurso de un cierto proceso mediante una trayectoria. De esta manera, se ve intuitivamente cómo diferencias muy pequeñas en los valores de las condiciones iniciales originan trayectorias que pueden alejarse muchísimo entre sí tras un tiempo relativamente corto. Así que las previsiones, además de ser solo probables, son fiables solo a corto plazo. Este modelo parece adecuado para tratar fenómenos como las previsiones meteorológicas y algunos otros, en los cuales la complejidad se reduce a la interferencia casual de una multiplicidad de factores que no se pueden prácticamente controlar y, en este sentido, nos muestra los límites de la metodología analítica que se basa en el programa de construir el resultado final mediante el conocimiento de los resultados parciales.

La teoría de sistemas, sin embargo, corresponde a otra perspectiva que se funda en una experiencia cognitiva muy común: las cosas que conocemos siempre nos aparecen como dotadas de ciertas propiedades y de una estructura propia que consiste en una red de relaciones bien precisas entre partes que están a su vez dotadas de propiedades y estructura. Si llamamos sistema a cualquier entidad caracterizada al mismo tiempo por ciertas propiedades y por una cierta estructura, podemos decir que la teoría de sistemas se basa en solo dos conceptos fundamentales, el de sistema y el de relación entre sistemas. Además, estos conceptos son fuertemente interdependientes en el siguiente sentido: las partes están ligadas entre sí por medio de relaciones mutuas y forman una unidad compleja, ordenada y dotada de individualidad propia, ya que se caracteriza por propiedades que son diferentes de las de las partes constituyentes (aunque dependen de ellas, en una cierta medida), tanto en sus propias características, como en su manera de funcionar.

Podríamos tal vez decir, más simplemente, que por sistema entendemos una totalidad ordenada de partes interrelacionadas cuyas características dependen tanto de las características de las partes como de la conexión específica entre ellas. Estas pocas palabras pueden expresar con más precisión el significado de la afirmación bastante común, pero vaga, de que "el todo es más que la suma de las partes". La perspectiva analítica intenta mostrar que, a final de cuentas, el todo se construye (o reconstruye) sumando los resultados del estudio de sus partes y, por consiguiente, tiene la misma naturaleza que sus partes (reduccionismo). La perspectiva sistémica, al contrario, reconoce las diferencias de propiedades y funciones de las diversas partes y, al mismo tiempo, afirma y subraya que el todo tiene propiedades suyas que dependen de las propiedades de las partes, pero que, gracias a ciertas relaciones entre las mismas, no pueden considerarse como un mero resultado de ellas. Se acostumbra a llamar holística -de hólos, en griego 'totalidad'a esta perspectiva que fue defendida especialmente por los teóricos del vitalismo, en el campo biológico y en oposición al mecanicismo, a finales del siglo XIX. Dicha perspectiva holística fue retomada de manera más rigurosa, evitando los aspectos más débiles del vitalismo, precisamente por un biólogo, Ludwig von Bertalanffy, fundador de la teoría de sistemas. ${ }^{3}$ Es natural que esta teoría se haya originado en el campo de la biología, ya que el organismo vivo es el ejemplo más claro y familiar de una entidad sistémica. Sin embargo, existe un número muy grande de entidades complejas en el mundo natural, social, histórico y cultural. Por ello, el enfoque sistémico se ha revelado muy fructífero en muchos ámbitos, como el mismo Bertalanffy ya había previsto en su época.

De manera especial, es un enfoque que ayuda a entender bien la enorme importancia que tiene la presencia de la tecnología en nuestro mundo actual. Es muy común entender que la creciente presencia de la tecnología en nuestras vidas consiste sobre todo en una difusión masiva de las máquinas, de artefactos materiales con los cuales es difícil convivir, aunque nos sirvan y creamos que estamos simplemente utilizándolos. En realidad, la tecnología es mucho más, es 
un verdadero sistema tecnológico, que tiene sus características, sus dinámicas, su autonomía, su tendencia a crecer sin límites. Así pues, nuestra civilización tecnológica es mucho más que una simple civilización de las máquinas. ${ }^{4}$ La bioética surge como respuesta a las situaciones inéditas a que nos han llevado los avances de las tecnologías biomédicas. Y, en función de lo dicho, tiene que ayudarnos a entender correctamente cuál es la relación de la ética con la tecnología, entendida esta como sistema.

\section{LA ÉTICA EN UN MUNDO TECNOLÓGICO}

Como acabamos de decir, los problemas bioéticos surgen de la aplicación de procedimientos tecnológicos particularmente avanzados en el campo de la vida y, en este sentido, ponen en tela de juicio la visión muy común según la cual el progreso tecnológico es de por sí positivo. Si fuese así, se podría aceptar la máxima de que "lo que se puede hacer tecnológicamente se debe hacer". En bioética, por el contrario, se debaten muchas situaciones en las cuales ciertas cosas se pueden técnicamente hacer, pero no se deben hacer por razones morales. Como estos no son casos aislados, sino que constituyen prácticamente la totalidad de las cuestiones específicamente bioéticas, podemos decir que la misma bioética aparece como caso emblemático de una reflexión epistemológica que intenta identificar un posible punto de contacto entre el progreso tecnocientífico y la evolución de la conciencia moral. Hablamos de evolución para indicar que no se trata de una revolución en la ética, sino de una necesaria madurez, exigida por la transición desde el ambiente natural, en el que se daba antaño la conducta humana, hasta el ambiente tecnológico en el que se da hoy día. Por consiguiente, podemos articular mejor la caracterización de la bioética que hemos mencionado al inicio y decir que la bioética es un diálogo crítico entre disciplinas con patrones epistemológicos diferentes, que trata de indicar normas para dirigir la acción individual y colectiva hacia la solución de conflictos que surgen de la aplicación de la tecnología a la vida.

Se trata, como hemos dicho, de una precisión que alcanzamos considerando un poco menos superficialmente en qué consisten los debates bioéticos concretos. Pero también llegamos a ella observando cuáles eran las inquietudes que se encontraban en el origen histórico de la bioética. Recordemos que dicho origen no coincide con el inicio de su desarrollo institucional en los años setenta del siglo pasado, cuando se fundaron el Hastings Center y el Kennedy Institute. Si adoptamos un punto de vista conceptual, como es obvio que debe hacerse, la creación del término bioética hace referencia a los escritos de Jahr, que no se ocupan en particular de ética médica, sino de un horizonte que concierne a la interrelación entre la acción humana y el conjunto de la naturaleza. Este encuentro entre progreso tecnológico y ética es la raíz profunda de la bioética, y nos invita a considerar como textos fundamentales en este sentido dos artículos raramente mencionados del teólogo católico Karl Rahner. ${ }^{5}$ Estos textos tratan con profundidad filosófica el tema de las manipulaciones genéticas, es decir de los primeros logros de la ingeniería genética, y plantean claramente el problema del control ético por parte del ser humano sobre los productos de la tecnología creada por el mismo hombre, con potenciales repercusiones sobre su propia identidad y naturaleza. Rahner no usa el término bioética, pero es significativo que en la misma época en la cual la bioética estaba a punto de imponerse culturalmente en los Estados Unidos, su horizonte ya abarcaba el ámbito de las biotecnologías y apuntaba, por tanto, muy claramente a la totalidad de los problemas que salían a la luz como exigencias de una ética a la altura de un mundo impregnado de tecnología.

\subsection{La aceptación de lo artificial}

Así concebida, la bioética se presenta como una respuesta al miedo hacia el progreso tecnológico que empezaba a manifestarse en los años '60 del siglo pasado, y que había producido en muchos autores y en muchos movimientos políticos un verdadero rechazo de la tecnociencia, sustituyendo al optimismo cientificista de la primera mitad del siglo. El programa bioético puede verse como la toma de conciencia del hecho de que este mundo tecnológico no es solamente el mundo en qué vivimos, sino el mundo del qué vivimos y que, por consiguiente, el verdadero problema consiste en aceptarlo y encontrar en él un lugar para la realización del ser humano. En este sentido, el hombre se siente solidario con el resto de la naturaleza, que se encuentra amenazada también por el desarrollo incontrolado de la tecnología. Medicina y biotecnología son los primeros ámbitos en los cuales este problema se manifiesta con claridad, pero abren un horizonte nuevo.

Un horizonte, en particular, en el cual pierde relevancia ética la oposición entre natural y artificial, según la cual la conformidad con la naturaleza representaba el criterio fundamental para juzgar moralmente las acciones humanas. Se trata de un antiguo principio enunciado por los filósofos estoicos y aceptado después por la ética cristiana. Según este principio, 
la naturaleza es obra de Dios y expresa su profunda sabiduría; por lo tanto, la acción del hombre es buena solo si se conforma a la naturaleza. Esta idea se puede aceptar sin condenar lo artificial en cuanto tal, sino advirtiendo que la capacidad de producir el mundo artificial de los artefactos y de las instituciones sociales es precisamente la expresión de la naturaleza humana en cuanto específicamente distinta de la pura y simple naturaleza animal. Por consiguiente, la desconfianza ante lo artificial no está vinculada necesariamente con el respeto hacia la naturaleza. Dicho respeto tiene que resultar compatible con un análisis lo suficientemente profundo y crítico de lo que es en realidad la naturaleza del hombre. Este problema, evidentemente, no puede resolverse considerando solo los aspectos biológicos de la vida, sino que requiere la consideración de muchas otras competencias de tipo humanístico en sentido amplio, así como, sobre todo, de la reflexión filosófica.

Estas últimas consideraciones sirven para evitar que nuestra "defensa" de lo artificial y de la tecnología pueda ser tomada como una justificación total de esta última. El ideal de la bioética como búsqueda de un punto de encuentro entre la tecnología y la conciencia moral se convertiría, así, en una visión de la bioética como instrumento para facilitar la aceptación social de la tecnología, para diluir los escrúpulos morales frente a tantas novedades tecnológicas que modifican profundamente las costumbres y los modelos de vida. Una perspectiva de este tipo no es ajena a los proyectos de las empresas industriales que impulsan las tecnologías más sofisticadas y las venden muy caras en el mercado, pero claramente no corresponde a la naturaleza propia de la bioética. Esta, en cuanto ética aplicada, tiene su razón de ser en la conciencia moral del hombre que, como ya hemos anotado, reconoce que entre las cosas que técnicamente se pueden hacer, hay algunas que moralmente no se deben hacer.

\subsection{El nuevo modelo epistemológico de la bioética}

Varios autores prefieren no considerar la bioética como una ciencia o como una disciplina científica en sentido propio, y prefieren designarla como un "campo de investigación", un "ámbito de problemas", un "conjunto de cuestiones" o algo de este tipo. La razón es que una ciencia, así como una disciplina particular dentro de una cierta ciencia, tiene que caracterizarse indicando cuál es el ámbito específico de sus objetos y cuáles son los criterios metodológicos para la realización correcta de su investigación y para la construcción de su conjunto específico de conocimientos. Es lo que a menudo se denomina "modelo epistemológico" de una determinada ciencia o disciplina. Si, como hemos visto, la tarea específica de la bioética es la de proponer normas o criterios de evaluación para la toma de una decisión éticamente correcta en situaciones complejas concretas, está bastante claro que estos juicios tendrán que tomar en cuenta los diferentes aspectos o factores que entran en la constitución de la situación misma, cada un uno de los cuáles lleva a considerar la situación desde un cierto punto de vista $y$, por consiguiente, a indicar una determinada solución para la toma de la decisión final. Por otro lado, cada punto de vista no se limita a ser la expresión de una cierta inclinación subjetiva, sino que suele acompañarse por la presentación de ciertos argumentos y criterios de comprobación que corresponden, en un sentido amplio, a otros tantos modelos epistemológicos de diferentes disciplinas.

Así las cosas, la tarea de la bioética parecería consistir en un análisis riguroso de los diferentes aspectos y factores de la situación, en una especie de mirada metateórica. No se ve, sin embargo, cómo de este análisis se puede pasar a una síntesis, en la cual consistiría lo específico de la bioética. La solución a esta dificultad se encuentra reconociendo que el modelo epistemológico de la bioética es el método interdisciplinar, un método que no es exclusivo de la bioética, sino que ha ido imponiéndose en varias disciplinas contemporáneas que tienen que ver con la complejidad. De hecho, la creciente demanda de bioética que constatamos en las sociedades actuales ha acabado con la desconfianza tradicional entre saberes diferentes en cuanto a sus patrones epistemológicos. Es más, con ello parece haberse inaugurado una nueva época de cooperación interdisciplinar, no solo entre las ciencias naturales y las ciencias humanas, sino también entre formas científicas y formas especulativas del conocimiento, como la filosofía y, en particular, la ética. Este efecto ha sido provocado por la complejidad de los objetos estudiados -ya lo hemos mencionado-, pero al mismo tiempo ha implicado un cambio de actitud intelectual, indispensable para alcanzar el nivel de intersubjetividad sin el cual no se da ningún conocimiento que pueda llamarse científico en sentido amplio. Este cambio de actitud, que podríamos calificar como la adopción de una categoría dialógica por parte del intelecto, es lo que constituye la raíz profunda del método interdisciplinar. Dicho cambio de actitud justifica también la búsqueda de una síntesis, que se realiza tras haber analizado debidamente la perspectiva y aportes objetivos de cada ciencia particular. 
No cabe dentro de los propósitos de este trabajo detenernos en la presentación, ni siquiera sumaria, de las características fundamentales del método interdisciplinar (que habría que distinguir de la simple multidisciplinariedad y de la transdisciplinariedad). Sin embargo, no podemos omitir otro aspecto de la bioética que tiene una cierta semejanza con la interdisciplinariedad, sin que llegue a confundirse con ella. Se trata del hecho de que en bioética es normal considerar no solo lo que nos dicen sobre un determinado problema diferentes perspectivas disciplinares (por ejemplo, médica, económica, psicológica, social, política, religiosa...), sino también las distintas soluciones propuestas por diferentes teorías éticas. En este caso, la actitud dialógica es mucho más difícil de adoptar, ya que los sujetos que participan concretamente en el debate están existencialmente comprometidos con una determinada doctrina ética, que es normalmente parte de su propia concepción de la vida, de la cual no es posible despojarse totalmente. Así, el análisis conceptual preciso, el examen riguroso de las argumentaciones, la indicación de consecuencias intuitivamente inaceptables o de incongruencias siguen siendo posibles pero no poseen la misma fuerza discriminatoria que pueden tener en las ciencias propiamente dichas. No obstante, la complejidad con la cual tiene que confrontarse la bioética tiene mucho que ver con esta pluralidad de las convicciones éticas presentes en nuestras sociedades, pluralidad destinada a aumentar con el imparable proceso de globalización y con las características cada vez más multiculturales que van asumiendo nuestras sociedades.

Esta también es una situación inédita con respecto no tanto a la ética médica como a la ética tradicional. Hasta el siglo XIX, podemos decir que dentro de cada gran área cultural existía una amplia base de normas morales comunes y aceptadas, y que las diversas éticas consistían en proponer los fundamentos o las justificaciones racionales de dichas normas a partir de diferentes principios generales. Hoy no es así: dentro de una misma sociedad existen conductas que muchos consideran moralmente lícitas y que otros consideran moralmente ilícitas. Como es evidente, solo teorías éticas diferentes pueden justificar estos puntos de vista opuestos. Lo que tenemos que reconocer respecto a la sociedad en general, vale, como bien se sabe, también en el campo de la bioética, en donde hay varias cuestiones de este tipo abiertas y debatidas. Hasta tal punto es así, que no es exagerado afirmar que precisamente la vivacidad y la actualidad de estos debates bioéticos ha despertado en las últimas décadas un verdadero renacimiento de la ética en cuanto tal.
Este hecho no debe sorprendernos, ya que el fin propio de las investigaciones y debates bioéticos consiste en indicar cuál sería la decisión correcta a propósito de un determinado curso de acción, lo cual significa reconocer explícitamente que el planteamiento metodológico y epistemológico de la bioética tiene que incluir claramente los elementos de una ciencia práctica en el sentido clásico, es decir de un saber orientado a dirigir la acción (cfr. Agazzi, 1992; Marcos, 2013).

\subsection{La dimensión holística de la ética}

Si la aceptación de lo artificial es una característica decisiva que distingue la bioética de la ética tradicional, cabe mencionar otra no menos profunda pero menos fácil de aclarar. Se trata del hecho de que, mientras la ética tradicional investiga las acciones del individuo, la bioética concierne especialmente a las acciones colectivas. Se trata de una transición que se ha producido paulatinamente, y de la que vamos a indicar algunos elementos esenciales.

En las sociedades pre-modernas, los efectos de las acciones del individuo caían en un entorno espaciotemporal limitado y podían considerarse como directamente producidos por él. Es decir, estos efectos eran previstos e intencionalmente perseguidos, de manera tal que no había discrepancia entre intención y efecto previsto de la acción. Entonces, el juicio moral podía basarse en la evaluación de la cualidad moral del efecto (bueno o malo). Solo si el efecto concretamente ocurrido resultara ser diferente de la intención o de la previsión podía cambiar el juicio moral sobre la acción misma. Con el inicio de la modernidad, la estructura de la vida social se hace mucho más compleja y la mayoría de las acciones de los individuos constituyen solo un segmento limitado de una red cuyo resultado final no depende de la intención del individuo, que ha realizado un pequeño segmento cuyos efectos a veces ni siquiera pueden ser previstos. De todas maneras, el individuo no puede controlar el resultado de su acción. Como consecuencia, el significado específicamente moral de las acciones termina por reducirse a la intención, que es necesariamente individual y subjetiva.

Hay también otras razones más profundas que podemos resumir en la centralidad que el pensamiento moderno ha reconocido al sujeto, pero nos llevaría demasiado lejos analizarlas. Será suficiente con apuntar que, en la ética más típica de la modernidad, la de Kant, el peso de la moralidad se concentra en la intención de la libre voluntad. "No hay 
nada absolutamente bueno en el mundo -afirma Kant- fuera de una voluntad buena". Se desprendía de esto que la responsabilidad moral es únicamente individual y se reduce al hecho de que el individuo tenga o no la intención de cumplir la acción de la cual se considera responsable (el discurso acerca de la responsabilidad económica o legal es totalmente diferente). De forma resumida, esta concepción se puede expresar en la máxima: "cumple con tu deber, pase lo que pase", es decir sin tomar en cuenta las consecuencias. Esta oposición entre una "ética de la conciencia" y una "ética de las consecuencias" se puede leer en Max Weber, para quien el concepto de responsabilidad (sobre todo en la vida política) se reduce a esto: alguien se declara responsable de sus acciones en el sentido de que está dispuesto a pagar él mismo el precio de sus consecuencias, es decir, que "se pone en juego a sí mismo", y nada más. No existe el sentido de una responsabilidad hacia alguien o hacia algo. ${ }^{6}$

Una ética inspirada en esta óptica no está a la altura de un mundo en el cual se reconoce fácilmente la presencia de acciones colectivas muy complejas que producen efectos concretos en general de gran tamaño y alcance, efectos que, en muchos casos, aparecen evidentemente como malos. Todas las realizaciones significativas de la tecnología son de este tipo y, vista la omnipresencia de este mundo tecnológico, es inevitable reconocer que la mayoría de las acciones que conciernen y afectan al mundo actual son precisamente acciones colectivas de este tipo. Por otro lado, no se ve cómo se podría atribuir una intención a un grupo de individuos e incluso a una red compleja de individuos que cooperan de hecho en las grandes producciones tecnológicas. ¿Diremos entonces que, faltando la intención, falta la base del juicio ético, o que nadie es responsable porque nadie en particular quiso realizar el efecto malo? Evidentemente no estamos dispuestos a aceptar estas conclusiones.

Para salir de la dificultad, tenemos que elaborar una ética en la cual la intención individual, aun sin ser descartada, desempeñe un papel reducido. Buscamos, pues, una ética que tenga en cuenta a varios actores, no en cuanto decisores libres, sino en cuanto portadores de ciertos valores, de ciertas competencias, de ciertas instancias, y todos ellos partícipes dentro de un determinado contexto y mutuamente relacionados según una red de interacciones más o menos eficaces. Esto significa que tenemos que considerar, como hace la perspectiva holística, unidades complejas que operan como un todo. El todo resulta de la cooperación eficaz de varias partes que son, por un lado, autónomas, y, por otro lado, no-independientes. La moderna teoría de sistemas nos ofrece el marco conceptual y también ciertos instrumentos técnicos para desarrollar esta perspectiva holística, dentro de la cual, en particular, se puede elaborar el concepto de responsabilidad compartida. Aquí la responsabilidad individual no desaparece, pero recibe un peso proporcionado a la importancia que el individuo tiene en el funcionamiento del sistema y en la toma de decisiones. Se trata de un discurso que todavía está en ciernes.

Cuanto acabamos de presentar podría, en un cierto sentido, enmarcarse dentro del modelo interdisciplinar del que ya hemos hablado. Pero hay algo más. En el mundo actual, como hemos ya anotado a propósito de la bioética, conviven multitud de posiciones éticas diferentes representadas por los distintos actores de las actividades complejas de la vida social. Sería un grave error tomar este hecho como pretexto para la aceptación de un relativismo ético. Dicho relativismo implicaría aceptar que en el campo ético "todo vale". Eliminaríamos así cualquier posible regulación ética del desarrollo de la sociedad tecnológica. Al contrario, la presencia de éticas diferentes indica que la ética no es prescindible. Y la ética se manifiesta a través de las diferentes posiciones morales, cada una de las cuales subraya y absolutiza un valor o principio ético importante. El problema, por lo tanto, consiste en hacer dialogar a estas diferentes teorías éticas, porque cada una ofrece un aporte valioso al madurar de una conciencia ética global más comprensiva. Nos damos cuenta, así, de cómo muchas teorías éticas expresan su pertenencia a tradiciones culturales que hoy confluyen gracias a la globalización. Por consiguiente, la actitud dialógica tiene que tratar de buscar una ética compartida para la sociedad del presente y del futuro, la cual de todas maneras tendrá que confrontarse globalmente con los tremendos retos que nos impone a todos el desarrollo de la tecnociencia.

\section{AGRADECIMIENTOS}

Este artículo fue redactado gracias al apoyo del Proyecto de Investigación EF-2012-33998 del Ministerio español de Economía y Competitividad. 


\section{NOTAS}

1. Cf. Treviranus, 1802; Lamarck, 1802.

2. No es difícil mencionar ejemplos. Según su etimología, geo-metría significa medición de la tierra y se relaciona con los conocimientos y las prácticas que los agrimensores del antiguo Egipto utilizaban para reconstruir los perímetros de los campos borrados por las inundaciones anuales del Nilo; pero ya con los griegos la geometría tenía el estatuto de una ciencia teórica pura, en la cual encontraba su aplicación más perfecta el método axiomático. Posteriormente se entendió como estudio de las diferentes figuras del espacio accesibles mediante una particular intuición; pero con el surgimiento de las geometrías no-euclidianas se afirmó la legitimidad de diferentes geometrías cada vez más abstractas, de modo que hoy se consideran como ramas de la geometría disciplinas matemáticas que apenas tienen dominios comunes. Pasando a la

\section{BIBLIOGRAFÍA}

Agazzi, E. (1992). Il bene, il male e la scienza. Milano: Rusconi.

Bernard, C. (1865). Introduction à l'étude de la médecine expérimentale. Paris: J. B. Baillière et fils.

Bertalanffy, L. von (1968). General System theory: Foundations, Development, Applications. New York: Braziller.

Ellul, J. (1977). Le système technicien, Paris: Calman-Levy

Jahr, F. (1927). Bio-Ethik. Eine Umschau über die ethischen Beziehungen des Menschen zu Tier und Pflanze. Kosmos. Handweiser für Naturfreunde, 24 (1), pp. 2-4. física, y limitando la atención a la época moderna, en la que se presentó como disciplina distinta de la filosofía natural, podemos decir que en una primera fase se identificó con la mecánica y la óptica, luego incluyó el estudio del calor y de los fenómenos electromagnéticos, para después abarcar el mundo subatómico, utilizando conceptos y teorías muy diferentes entre sí e incluso a veces casi incompatibles. Lo que, a pesar de todo, permite considerar estas ciencias como suficientemente definidas es su estructura epistemológica; por ello podemos considerar como legítimo el propósito de hacer algo parecido también en el caso de la bioética.

3. Bertalanffy empezó a desarrollar sus ideas en la dirección de la teoría de sistemas en los años treinta del siglo pasado, pero publicó su obra más completa sobre este tema muchos años más tarde (Bertalannfy, 1968).

Lamarck, J.-B. (1802). Hydrogeologie. Paris: Museum d'histoire naturelle.

Marcos, A. (2013). Ciencia y acción. Una filosofía práctica de la ciencia. México: Fondo de Cultura Económica.

Mori, M. (2002). Bioetica. 10 temi per capire e discutere. Milán: Mondadori.

Mumford, L. (1967). The Myth of the Machine (first vol.). New York: Harcourt Brace.

Poincaré, H. (1890). Sur le problème des trois corps et les équations de la dynamique. Acta Mathematica, 13 (1), pp. A3-A270.
4. Este enfoque está bien presentado en el libro de Jacques Ellul (1977), mientras que la famosa obra de Lewis Mumford (1967) seguía aceptando el patrón de la civilización de las máquinas.

5. Estos dos artículos son Experiment Mensch (El hombre como objeto de experimentos) y Zum Problem der genetischen Manipulation (El problema de la manipulación genética). El primero es el texto de una conferencia dada en diferentes países entre 1965 y 1967, y el segundo es una contribución publicada en un volumen colectivo en 1968. Ambos se pueden encontrar en Rahner (2001), respectivamente en las páginas 437-456 y $498-524$.

6. Weber propuso estas ideas en su conferencia titulada "La política como profesión", leída en Zurich en 1919. El texto se puede encontrar en Weber (1992).

Potter, R. (1971). Bioethics: Bridge to the Future. New Jersey: Prentice Hall

Rahner, K. (2001). Verantwortung der Theologie: Im Dialog mit Naturwissenschaften und Gesellschaftstheorie. Freiburg i.Br.: Herder

Treviranus, G. R. (1802). Biologie oder Philosophie der lebenden Natur für Naturforscher und Aerzte. Göttingen: Johan Friedrich Röwer

Weber, M. (1992). Politik als Beruf (Vortragsmitschrift mit Nachwort von Ralf Dahrendorf). Stuttgart: Reclam. 